\title{
Vogons, the Invaders
}

\author{
Pedro Henrique dos Santos Kutni \\ Instituto Federal Catarinense \\ p.henriquekutni@hotmail.com \\ Vinícius Feldhaus Gobetti \\ Instituto Federal Catarinense \\ vinigs2.gobetti@gmail.com
}

\author{
Gustavo Danielevig Pereira Dias \\ Instituto Federal Catarinense \\ gustavo_dpdias@hotmail.com
}

\author{
Adriano Pessini \\ Instituto Federal Catarinense \\ adriano.pessini@ifc.edu.br
}

\author{
Josef $M$ athaus Bischof \\ Instituto Federal Catarinense \\ theobischof@hotmail.com
}

\author{
Luiz Ricardo Uriarte \\ Instituto Federal Catarinense \\ luiz.uriarte@ifc.edu.br
}

\begin{abstract}
ABST RACT
Game development is a recognized and documented approach to improving computer programming learning. This article describes the redesign of the popular game Space Invaders, using Python as the programming language and libraries Arcade, Random, Os and Math. The game developed has modifications in various aspects, like textures, jogability, scores and story. The motivation for the remake was the desire to bring a nostalgic experience for the player, combined with learning about game development. Considering that it was built by people with no previous experience in game development, the proposed objectives were achieved, with the game having been completed. The Arcade library proved to be easy to use and productive. As future work, an online ranking will be developed, in addition to generating bonuses for the player and the use of design patterns.
\end{abstract}

\section{KEYWORDS}

Python, Arcade, Desenvolvimento De Jogos

\section{Introdução}

Há bastante tempo os jogos são uma diversão e um entretenimento para as pessoas do mundo, independente da faixa etária, pois existem jogos de estilos variados para os mais diversos gostos pessoais, destacando-se, entre eles, os jogos eletrônicos. Os jogos eletrônicos se tornaram populares a partir da década de 70/80, e com isso muitos jogos do gênero arcade também ficaram muito conhecidos, como, por exemplo: Ms. Pacman, Donkey Kong e Aero Fighters [1].

O desenvolvimento de jogos eletrônicos sempre contou com programadores para desenvolver os códigos que dão a vida para o jogo. Com o avanço das linguagens de programação e engines de desenvolvimento de jogos, o seu desenvolvimento se tornou mais simples e permitiu que fosse possível a criação de um jogo feito por amadores da área da programação [2].

Com o mercado mundial de jogos digitais se tornando um dos mais lucrativos dos últimos tempos [4], houve uma busca para aplicar algum redesign em jogos populares do século passado, alterando elementos gráficos ou sua jogabilidade. Inspirandose no jogo "Space Invaders", foi aplicado esse intuito de redesign e houve o começo da criação do jogo "Vogons, the invaders". O presente trabalho está dividido em 4 seções: na primeira é metodologia usada no desenvolvimento do jogo; a segunda apresenta o desenvolvimento do jogo; a terceira seção apresenta os resultados obtidos com esse desenvolvimento e na quarta seção apresenta-se as considerações finais do trabalho.

\section{Metodologia}

Na primeira etapa houve a pesquisa de maneiras viáveis para se desenvolver um jogo, sendo que ao longo do estudo foi escolhido o Python como linguagem de programação por conta do seu grande crescimento nos últimos anos e sua simplicidade para leigos na área

[3].

$\mathrm{Na}$ segunda etapa, inspirado no redesign do jogo em "Space Invaders", foi elaborado o projeto do jogo onde foram estabelecidas informações relevantes como: design do jogo, temática, personagens, enredo, entre outros. Também foi decidido pelo uso da biblioteca de criação de jogos Arcade, pois a mesma tem uma ótima usabilidade, funções prontas e com todos os atributos para o desenvolvimento de um jogo.

A terceira etapa se baseia no estudo do Python juntamente com as bibliotecas Arcade, Random, Os e Math, onde buscou-se o aprendizado e aperfeiçoamento nos mesmos.

A quarta etapa foi destinada à criação da arte do jogo através do AsePrite (editor de Pixel Art) e Photoshop (editor de imagens) e a codificação do jogo foi realizada usando o editor de textos

\section{Desenvolvimento}

$\mathrm{O}$ código do jogo foi feito inteiramente orientado à objeto, com o desenvolvimento das classes demonstradas na Figura 1 que contém toda a lógica de sua funcionalidade, sendo elas: Jogo, Tiro, Nave, Base, Nave_Inimiga e Boss.

Estas classes são especializações da classe Sprite do Arcade que provê recursos de manipulação dos sprites.

Na Figura 1 pode ser observada a imagem das naves utilizadas no jogo, sendo nave inimiga (em 1), Boss (em 2) e a nave do jogador (em 3). As imagens foram elaboradas pelos autores, seguindo as cores e a temática do jogo.

Conforme pode ser observado na Figura 2, a execução de um jogo respeita duas etapas bem definidas: a inicialização e a lógica de jogo, também conhecida como game looping. 


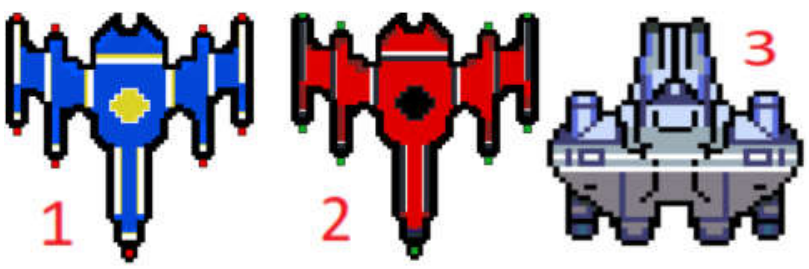

Figura 1 - Naves do jogo. Fonte: A utores, 2019.

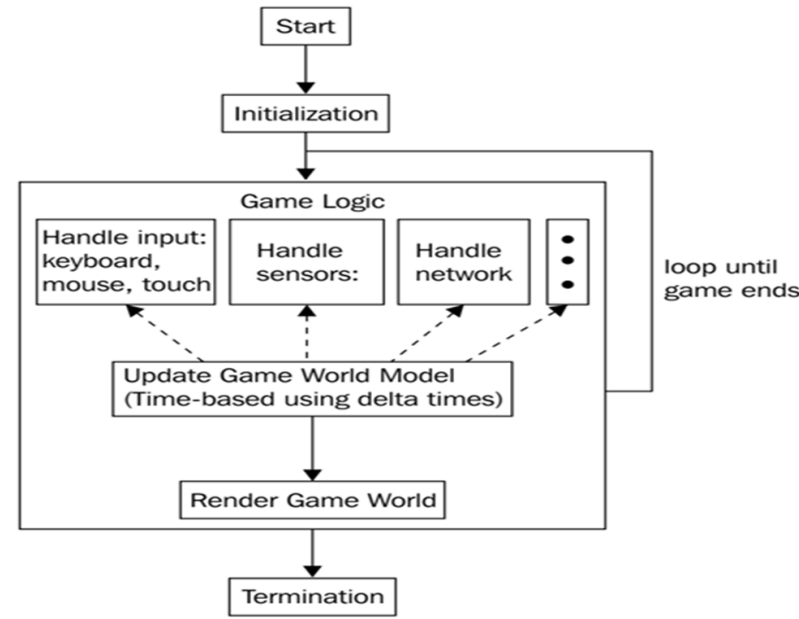

Figura 2 - Processo de inicialização de um jogo. Fonte: N air e Oehlke, 2015. [5].

$\mathrm{Na}$ etapa de inicialização, o programador deve realizar o carregamento das texturas, animações e áudios, o posicionamento do jogador e do jogo e a definição do estado inicial do jogo. No Arcade, a inicialização é realizada por meio da implementação de um método chamado setup(), conforme pode ser observado na Figura 3.

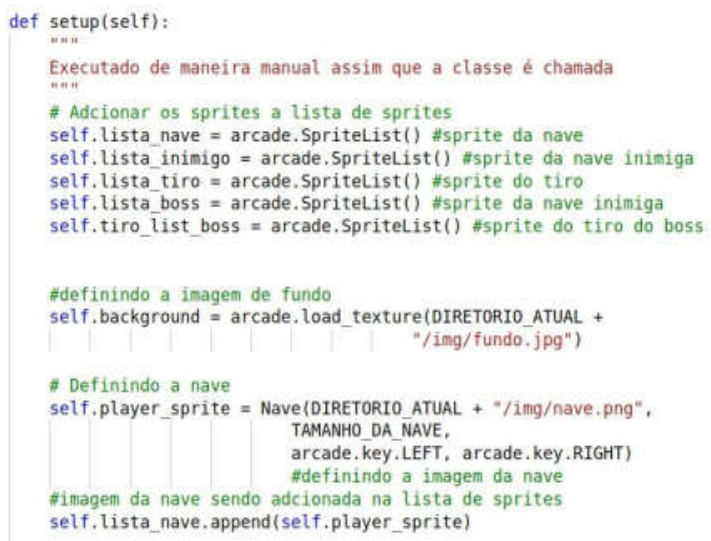

Figura 3 - Carregamento de textura utilizando Arcade. Fonte: Autores, 2019.

A lógica do jogo (também chamada de game looping) é programada por meio da implementação do método update() que, quando chamada, é executada automaticamente. Neste projeto, este método foi usado para funções como: a movimentação do jogador, controlador da pontuação, spawn da nave inimiga, entre outros.

A Figura 4 mostra um trecho da codificação do jogo, essa parte do código é responsável por fazer a geração aleatória dos inimigos no topo da tela de jogo, porém limitando a posição no eixo Y em 400 pixels para garantir uma distância de segurança para o jogador. Para fazer a geração aleatória foi usada a biblioteca Random, onde a mesma gera números aleatórios entre aqueles definidos na codificação que são usados no posicionamento dos inimigos nos

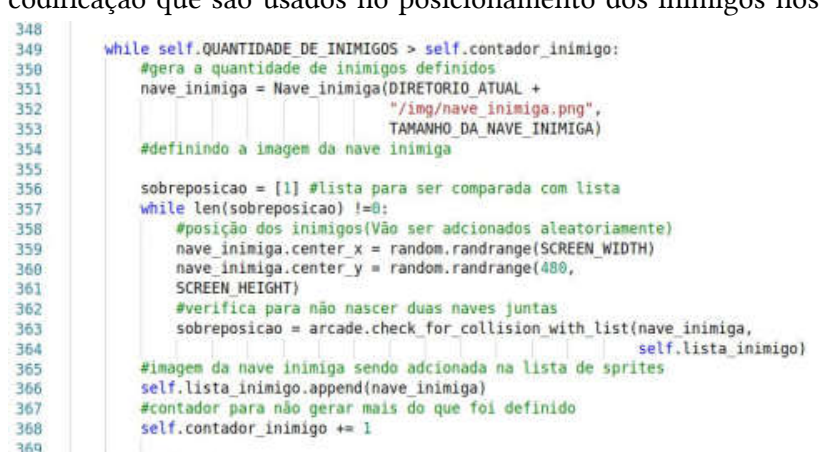

Figura 4 - Geração de inimigos na tela. Fonte: Autores, 2019.

Na Figura 5 mostra-se como é feito a mira do tiro do boss, cujo propósito é sempre seguir o jogador. Para se fazer essa "mira guiada" foram utilizados conhecimentos matemáticos, no caso o arco tangente do coeficiente dos parâmetros sendo eles o eixo x e y (ângulo teta).

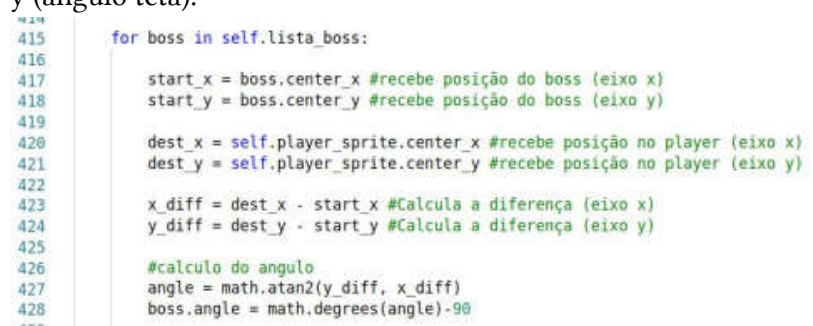

Figura 5 - Implementação da "mira guiada". Fonte: Autores, 2019.

A movimentação da nave inimiga é feita na sua própria classe, como pode ser observado na figura 6. A movimentação da nave inimiga é feita somente no eixo $\mathrm{Y}$, com uma velocidade de -1 pixel por frame.
12
def update(self):
13 self.center $y=1$

\section{Figura 6- Codificação do movimento da nave inimiga.} Fonte: Autores(2019)

Já a movimentação do jogador é feita em dois momentos para separar as atribuições e responsabilidades de acordo com os princípio da orientação a objetos: primeiramente na classe Jogo (Figura 7) e logo após na classe Nave (figura 8), pois 
das teclas de movimentação pressionadas pelo jogador é realizada na segunda, enquanto que a primeira agrupa as funcionalidades relacionadas à lógica do jogo que envolvem a nave do jogador, como por exemplo a verificação de colisão, entre outros.

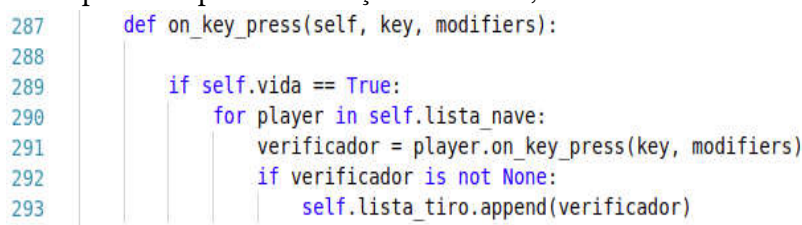

Figura 7 - Movimentação do player na classe Jogo. Fonte: Autores, 2019.

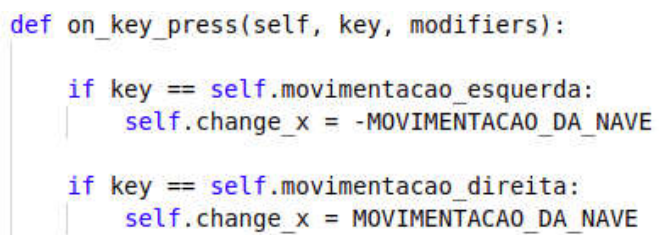

Figura 8 - Movimentação do player na classe Nave. Fonte: Autores, 2019.

\section{Resultados obtidos}

Com o fim da codificação os resultados obtidos foram além do esperado, levando em consideração que foi construído por pessoas sem experiência no desenvolvimento de jogos. Confira abaixo as telas do jogo "Vogons, the invaders" em execução:

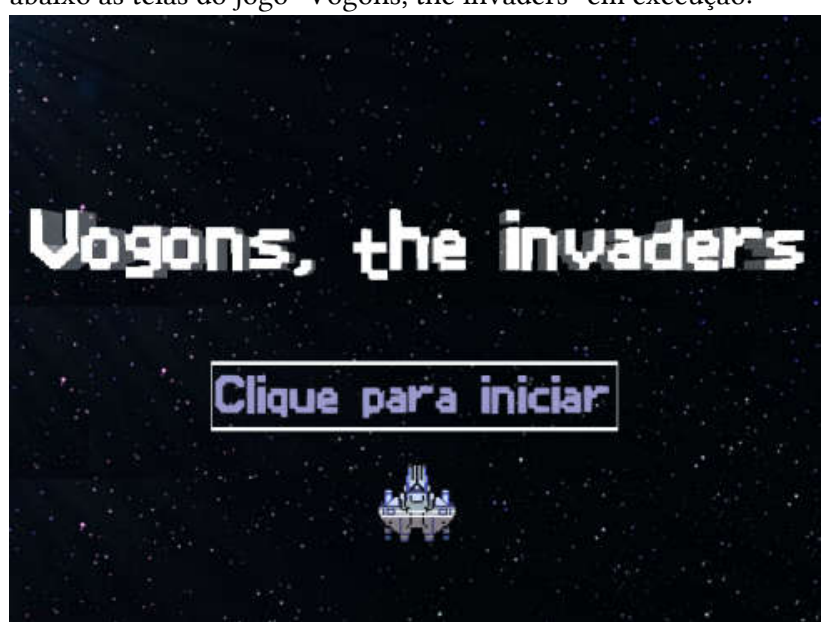

Figura 9 - Tela inicial do jogo. Fonte: Nair e Oehlke, 2015.

A tela de jogo é exibida na Figura 10, onde podem ser observados o tempo de jogo (em 1), a quantidade de disparos disponíveis para o jogador (em 2), a quantidade de hordas de inimigos que já foram geradas, o inimigo da classe Nave_Inimiga (em 4), o boss com o disparo guiado na direção do jogador (em 5), a nave do jogador (em

6), a imagem da base com o respectivo percentual de durabilidade restante (em 7, que se chegar a zero causa o encerramento por perda do jogo), a pontuação alcançada pelo jogador (em 8) e o

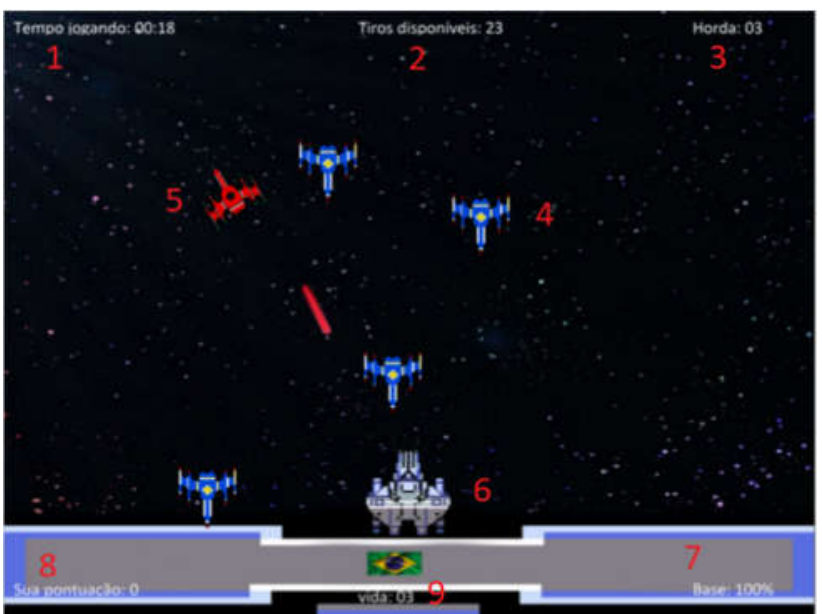

Figura 10 - Jogo em execução. Fonte: Autores, 2019.

\section{Considerações finais}

Diante do exposto, o presente trabalho mostrou o desenvolvimento de um jogo usando a linguagem de programação Python. O objetivo deste trabalho foi o enriquecimento de conhecimento pessoal na área da informática, mais precisamente em programação. A utilização da biblioteca Arcade proporcionou facilidade no desenvolvimento do jogo, sendo produtiva e trazendo diversão no decorrer do desenvolvimento do mesmo. Recomenda-se a utilização desta biblioteca para os iniciantes na área de programação, uma vez que é de fácil compreensão e acessível a todos. Vale observar que, apesar de ser de fácil utilização, o desenvolvimento de sistemas não deve se limitar a uma única biblioteca, visto o constante desenvolvimento da área de informática, principalmente no que diz respeito às ferramentas para produção de jogos. Como trabalhos futuros, estão previstas implementações de um ranking online dos jogadores, a geração de bônus para os jogadores que aumentem o número de vidas, velocidade, quantidade de disparos, entre outros. Outra possibilidade, como recomendação para trabalhos futuros, e a utilização de padrões de projeto durante o desenvolvimento.

\section{REFERÊNCIAS}

[1] Batista, M. L. S.; Quintão, P. L.; Lima, S. M. B.. Campos, L. C. D.,Batista, T. J. S. Revista Eletrônica da Faculdade M etodista Granbery, n 3,jul/dez 2007. [2] Tiobe (2019) "TIOBE Index for October 2019", https://www.tiobe.com/ tiobeindex/, Outubro 2019

[3] Valente, J. A . e A Imeida F. J. Visão analítica da informática na educação no Brasil: A questão da formação do professor. Revista Brasileira de Informática na Educação - Número 1 - 1997.

[4] Ximenes, M.,, Souza, L. J., Malcher, F., Marinho, L., Neves, A. eCampos, F. Redesign de Jogos Clássicos VII Brazilian Symposium on Computer Games and Digita Entertainment November, 10-12, 2008

[5] Nair, S. B. e Oehlke, A. Learning LibGDX Game Development. Second Edition-Packt Publishing, 2015 